\title{
Modelling Oscillator Synchronisation During Vertebrate Axis Segmentation
}

\author{
Philip J. Murray Philip K. Maini, and Ruth E. Baker
}

\begin{abstract}
The somitogenesis clock regulates the periodicity with which somites form in the posterior pre-somitic mesoderm. Whilst cell heterogeneity results in noisy oscillation rates amongst constituent cells, synchrony within the population is maintained as oscillators are entrained via juxtracine signalling mechanisms. Here we consider a population of phase-coupled oscillators and investigate how biologically motivated perturbations to the entrained state can perturb synchrony within the population. We find that the ratio of mitosis length to clock period can influence levels of desynchronisation. Moreover, we observe that random cell movement, and hence change of local neighbourhoods, increases synchronisation.
\end{abstract}

\section{Introduction}

Somitogenesis is the process by which the pre-somitic mesoderm (PSM) segments at periodic time intervals into regularly spaced blocks of epithelial cells. The frequency at which new pairs of somites are formed is regulated by the somitogenesis clock, a population of coupled molecular oscillators that is present in the posterior PSM, while a travelling front determines the position of somite formation.

Notch-Delta signalling is a key cell-cell communication mechanism in the PSM $[1,2]$ and it is thought that one of its roles is to synchronise the oscillations of

P.J. Murray • R.E. Baker

Centre for Mathematical Biology, Mathematical Institute, University of Oxford, 24-29 St Giles', Oxford OX13LB, UK

e-mail: murrayp@maths.ox.ac.uk; ruth.baker@maths.ox.ac.uk

P.K. Maini $(\bowtie)$

Centre for Mathematical Biology, Mathematical Institute, University of Oxford, 24-29 St Giles', Oxford OX1 3LB, UK

e-mail: maini@maths.ox.ac.uk 
neighbouring oscillators [3]. However, mutants for different Delta ligands are observed to affect oscillation patterns in the PSM in different ways, suggesting more than the single role of synchronisation in the PSM for Notch-Delta signalling [4, 5], or at the very least a complex interaction between Notch-Delta signalling components and the somitogenesis clock.

Cell movement in the mouse PSM has recently been quantified: there is a posteriorly increasing motility gradient along the anterior-posterior (AP) axis [6]. This random cell motion results in cells changing their neighbours, at least in the posterior tip of the PSM which, in turn, could influence the synchronisation of neighbouring cells. Random cell movement has also been quantified in zebrafish [1], although spatial variation in motility rates along the AP axis has not, to the best of our knowledge, been documented. A recent computational study has indicated that random cell movement should increase synchronisation in a population of locally coupled oscillators [7].

Synchronisation within the PSM is also perturbed by mitosis: when a cell enters $\mathrm{M}$ phase of the cell cycle it ceases the transcription necessary to progress its somitogenesis clock $[3,8]$. Thus, when the cell divides both mother and daughter cells are out of phase with their neighbours. To the best of our knowledge, the importance of mitotic perturbations in the maintenance of oscillator synchrony has not been quantified.

There is a long history of modelling of somitogenesis (see [9], for a review). Recent models have tended to utilise the increasing knowledge of molecular regulation of the clock (e.g. [10-14]). However, given incomplete knowledge of the governing molecular networks, the seemingly intricate relationship between oscillator coupling and the molecular clocks, and the difficulty in unambiguously parameterising the current models, coarse-grained descriptions of molecular oscillators have recently been considered in which progression through the somitogenesis clock cycle is described by a single variable - the oscillator phase [15-17].

In this study we consider a phase description of a population of locally coupled oscillators and investigate how population synchrony is perturbed by both cell movement and mitosis. The layout is as follows: in Sect. 2 we introduce a spatially distributed population of coupled oscillators and describe how synchrony can be measured in the population; in Sect. 3 we introduce a perturbation representing cell proliferation and examine the effects on oscillator synchrony; in Sect. 4 we allow random movement within the population and again consider the effects on oscillator synchrony; and, finally, in Sect. 5 we conclude with a discussion on how these results are relevant to our understanding of somitogenesis.

\section{A Simple Model for Cell Synchronisation in the PSM}

As we are primarily interested in studying the synchronisation of oscillators in the context of the PSM, we consider a cellular automation (CA) of size $30 \times 6$ cells, which is approximately of the same proportion as the zebrafish PSM. We define 
$\theta_{i}(t)$ to be the phase of the oscillator at time $t$ and assume that the governing phase dynamics are given by

$$
\dot{\theta}_{i}=\omega+\sum_{j} A \sin \left(\theta_{j}-\theta_{i}\right) ; i=1: N
$$

where $A$ is the strength of diffusive coupling, $\omega$ is the oscillator frequency, the sum is taken over nearest neighbours on the CA lattice and $N$ is the number of cells in the simulation. We note that the phase description of a population of coupled oscillators can be derived from an underlying molecular description with the sinusoidal coupling term representing the leading order term in a more general coupling function [18].

In order to quantify synchronisation in the oscillator population we define the global order parameters $r(t)$ and $\Psi(t)[18]$ such that

$$
r e^{i \Psi}=\frac{1}{N} \sum_{j=1}^{N} e^{i \theta_{j}}
$$

When $r=0$ the oscillators are completely out of phase while $r=1$ corresponds to global synchronisation. The variable $\Psi$ represents the mean phase of the population so when $r=1, \Psi=\omega$.

\subsection{Results}

In order to investigate perturbations from the spatially homogeneous phase distribution typically observed in the posterior PSM, we simulated populations of oscillators with phase dynamics given by (1) in which the initial phase of each oscillator was uniformly selected from the range $[0, \chi]$. Typically we choose $\chi \sim 1$ to represent a small initial perturbation from global synchrony. In Fig. 1 we present snapshots of phase distributions at $t=0$ and $t=3 \mathrm{~min}$, respectively.

The well-studied effect of nearest-neighbour sinusoidal coupling is to synchronise initially out-of-phase oscillators. As $t \rightarrow \infty$, the phase differences between oscillators tends to zero, and each oscillator oscillates with natural frequency, $\omega$. Calculating the order parameter (see Fig. 2) allows us to quantitatively measure global synchrony in the population as time evolves. As an aside we note that it may be feasible in the future to track in real time the phase of individual oscillators using reporter genes. If so, the order parameter could be measured by relating the activity of the reporter to a nominally defined 'phase' and using (2) to measure global synchrony. The effects of perturbations to global synchrony, such as the qualitative experiments performed by Horikawa et al. [3], could then be measured experimentally and the resulting order parameter data could be directly compared with theoretical models, such as (1). 

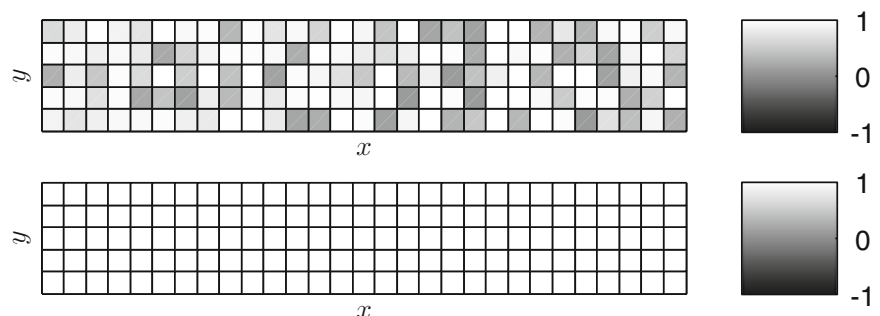

Fig. $1 \operatorname{Sin}\left(\theta_{i}\right)$ is plotted for each of the $N$ cells on the CA lattice. Top: the initial phase distribution in a typical simulation $(\chi=1)$. Bottom: the spatially uniform phase distribution at $t=3$. Cell phases on the CA lattice were updated using (1). Parameter values as in Table 1

Table 1 Parameters used in the simulations

\begin{tabular}{llll}
\hline Parameter & Value & Unit & Source \\
\hline$A$ & 2.2 & $\min ^{-1}$ & Murray et al. [16] \\
$\omega$ & 0.2 & $\min ^{-1}$ & Giudicelli et al. [19] \\
$T$ & 240 & $\min$ & Kane [20] \\
$M$ & $>15$ & $\min$ & Horikawa et al. [3] \\
\hline
\end{tabular}

${ }^{\text {a }}$ Parameter estimated from previous model

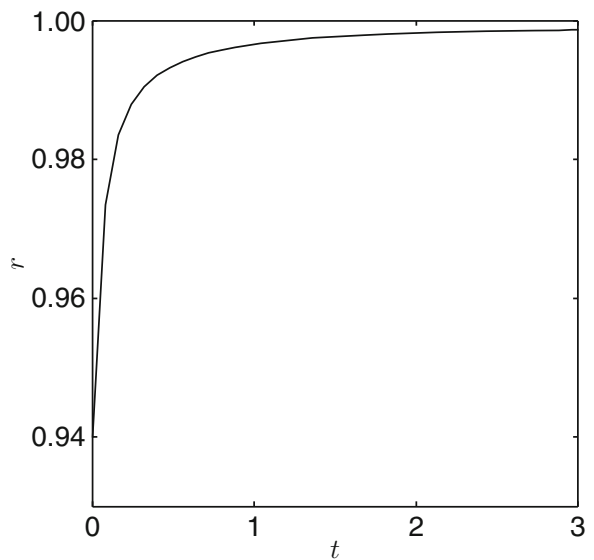

Fig. 2 The order parameter, $r$, is plotted against time, $t$. Cell phases on the CA lattice were updated using (1) and the order parameter was calculated using (2)

\section{Investigating the Perturbation to Oscillator Synchronisation Arising from Cell Mitosis}

Our goal in this study is to investigate how biologically-motivated perturbations to a population of sinusoidally coupled phase oscillators can influence phase synchronisation. We now examine if perturbations arising from mitosis can 
significantly destabilise oscillator synchrony within physiologically relevant parameter ranges.

\subsection{Model Development}

Using zebrafish, Horikawa et al. [3] observed that cells which were either in, or had just recently exited, $M$ phase of the cell cycle tended to be out of phase with neighbouring oscillators in the posterior PSM. They concluded that during M phase of the cell cycle the transcription necessary to progress the somitogenesis clock was paused as the cells prepared for division, thus dividing cells drifted out of phase with their neighbours. After $M$ phase the cells re-initiated the transcription necessary for somitogenesis clock progression, and, via cell-cell coupling, resynchronised with their neighbours. It was estimated that over a time observation window of one somitogenesis clock cycle ( $30 \mathrm{~min}), 10-15 \%$ of cells had either undergone or were undergoing $\mathrm{M}$ phase of the cell cycle, which was thought to last for a minimum of $15 \mathrm{~min}$.

In order to study the effect of this phenomenon on a two-dimensional lattice, we consider a modified equation for the phase dynamics given by

$$
\dot{\theta}_{i}=\left(\omega+\sum_{j} A \sin \left(\theta_{j}-\theta_{i}\right)\right) H\left(T-M-a_{i}\right),
$$

where $a_{i}$ is the age of the $i$ th cell, $T$ the cell cycle period, $H($.$) represents the$ Heaviside function and $M$ represents the time spent in $\mathrm{M}$ phase of the cell cycle. Cell age increases proportionally with time and is reset to zero when $t=T$. When a cell's age lies in the range $a_{i} \in[T-M, T]$ it is in M phase of the cell cycle and does not update its somitogenesis clock. We note that when a cell reaches the end of the cell cycle we do not introduce a daughter cell into the simulation. This simplification allows us to investigate computationally the effect of mitosis on phase synchronisation, without having to account for processes such as growth and apoptosis and the many more parameters they would introduce into our model.

\subsection{Results}

We performed a range of simulations with different values of the parameters $A$ and $M$. The initial phase distributions were as described in Sect. 2.1 while the initial cell cycle position for a given cell was chosen randomly from the uniform distribution $[0, T]$. In Fig. 3a the phase distribution is plotted at $t=240$ while in Fig. $3 b$ cells in $\mathrm{M}$ phase of the cell cycle are highlighted. Note the correlation between a cell being in $\mathrm{M}$ phase and being out of phase with its neighbours. The result of the mitosis- 


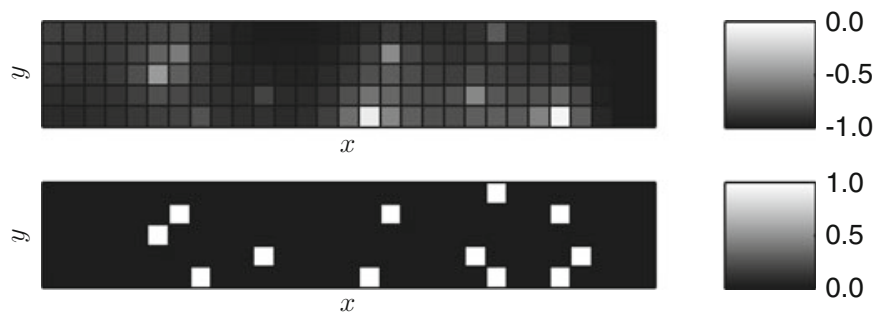

Fig. 3 Phase (top) and cell cycle (bottom) distributions at $t=4 \mathrm{~h}$. Cell phases on the CA lattice were updated using (3)

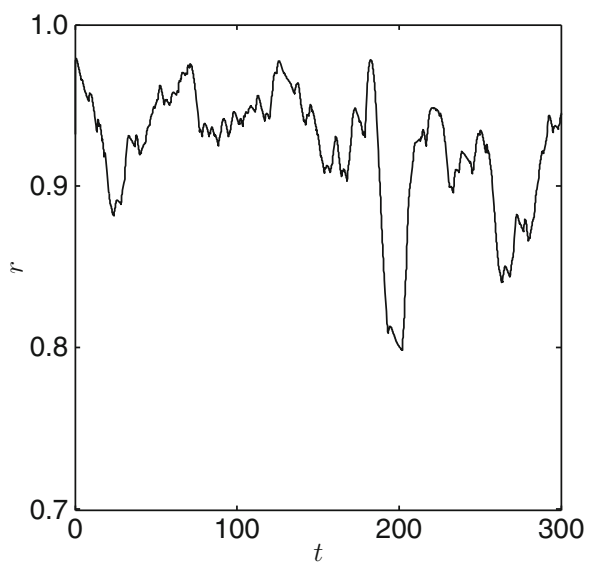

Fig. 4 The order parameter, $r$ is plotted against time. Cell phases on the CA lattice were updated using (3) and the order parameter was calculated using (2). Parameter values as in Table 1

induced clock-stopping perturbation is that the cell population does not attain global synchrony (see Fig. 4).

In Fig. 5 we plot the time-averaged global order parameter for a range of different values of the parameters $A$ and $M$. For a fixed value of the parameter $A$, the global order parameter firstly decreases and subsequently increases with increasing $M$. This biphasic behaviour can be explained by consideration of the period of the somitogenesis clock ( $30 \mathrm{~min})$ and the length of time spent in M phase ( $\sim 15 \mathrm{~min})$. As $M \rightarrow 0$ the somitogenesis clock is paused for increasingly shorter amounts of time and the perturbation to global synchrony is negligible. As $M$ increases, dividing cells pause their somitogenesis clocks for longer times, and thus move out of phase with neighbouring cells; hence global synchrony decreases. However, as $\mathrm{M}$ increases further neighbouring non-dividing cells are almost a full somitogenesis clock cycle ahead of the cell that entered $M$ phase when it returns to updating its somitogenesis clock. Thus the order parameter increases for larger values of $M$. The simulation results suggest that there is an optimal (in terms of phase desynchronisation) ratio of the parameters $M$ and $\omega$. One might 
Fig. 5 The time-averaged order parameter plotted against $A$ and $M$. For different parameter values cell phases on the CA lattice were updated using (3) and the time-averaged order parameter was calculated by averaging over (2)

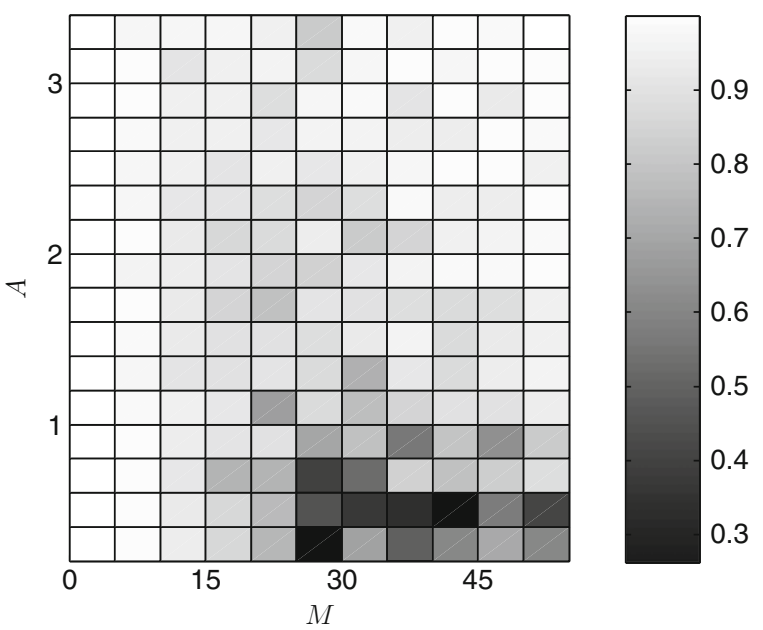

expect the optimal ratio would be such that the time spent in mitosis was half the somitogenesis clock period. However, the simulation results suggest that the optimal length of $\mathrm{M}$ phase is longer than half the somitogenesis period. This occurs because when an oscillator pauses its somitogenesis clock for M phase of the cycle, its neighbours, which are updating their phase, continue to be influenced by it and the effect of the growing phase difference is to slow their effective oscillation rate.

\section{Investigating the Perturbation to Oscillator Synchronisation Arising from Random Cell Movement}

Bénazéraf et al. [6] have recently quantified random cell motion in the mouse PSM while, in a theoretical study, Uriu et al. [7] have demonstrated that random cell movement enhances synchronisation of neighbouring molecular oscillators. We now investigate the effects of random cell movement on the mitosis-perturbed phase dynamics introduced in Sect. 3.

\subsection{Model Development}

In order to simulate random cell movement on the CA lattice, at each time step of a simulation we make $N$ random selections of a cell from the population and perform a swap with a randomly chosen neighbour with probability $p$. The phase dynamics are updated using (3). Thus the cellular automaton simulations are used to investigate the interaction between three key processes in the PSM: (a) phase oscillations and synchronisation; (b) perturbation to synchronisation due to mitosis; and (c) random cell movement. 


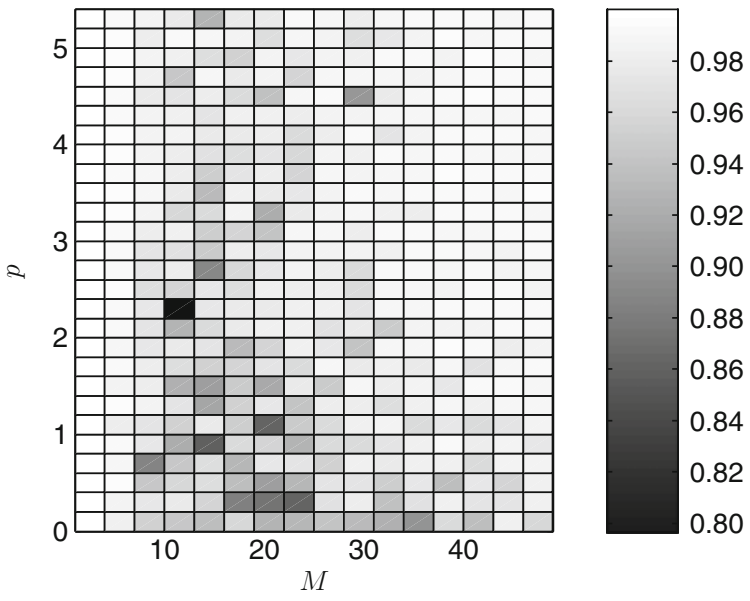

Fig. 6 The time-averaged order parameter plotted against $p$ and $M$. For different parameter values cell phases on the CA lattice were updated using (3) and the time-averaged order parameter was calculated by averaging over (2). Cells were allowed to randomly swap positions with their neighbours with probability $p$

\subsection{Results}

In order to quantify the effects of random cell movement on global synchrony, we performed simulations in biologically relevant regions of parameter space and measured the time averaged global order parameter. Firstly, we held the coupling strength, $A$, fixed and varied the random cell movement and the mitosis-induced perturbation considered in the previous section. For small values of $p$, and hence little random movement, the order parameter has a minimum, as in Fig. 5, for some intermediate value of $M$ (see Fig. 6). However, as $p$ increases and cells interchange neighbours more frequently, global synchronisation in the simulations increases. Thus random cell movement can counteract the reduction in order parameter resulting from the perturbation due to mitosis.

Next we held the time spent in mitosis fixed at $M=15 \mathrm{~min}$, the lower bound reported by Horikawa et al. [3], and examined how global synchrony in the simulation varied with the coupling strength and random movement (see Fig. 7). The order parameter is clearly, on average, lower in the bottom left corner of the figure, i.e. when the coupling strength and random cell movement rate are low. A low coupling strength can be compensated for by increasing the rate of random movement and vice-versa. This result is of particular interest as the two mechanisms enhancing synchrony in the simulations represent two completely different biological mechanisms: the coupling strength $A$ represents the effect of biochemical cell-cell signalling while the parameter $p$ represents a mechanical interaction. In zebrafish cell-cell coupling is strongly dependent on the NotchDelta signalling pathway while in chick it has been shown that cell motility is strongly influenced by an Fgf gradient along the AP axis [6]. 


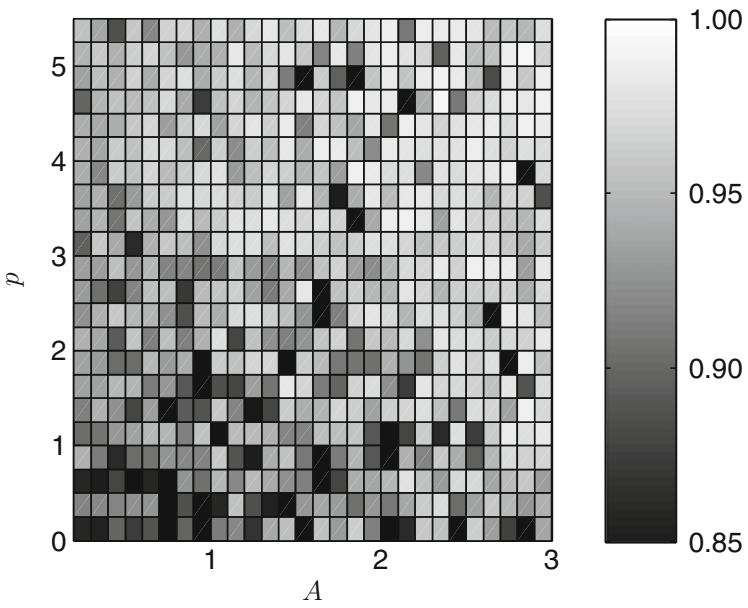

Fig. 7 The time-averaged order parameter plotted against $A$ and $p$. For different parameter values cell phases on the CA lattice were updated using (3) and the time-averaged order parameter was calculated by averaging over (2). Cells were allowed to randomly swap positions with their neighbours with probability $p$

\section{Discussion}

The phenomenon of oscillator synchronisation plays an important role in the posterior PSM during somitogenesis: as a result of cell heterogeneity neighbouring cells have different natural frequencies and without some coupling mechanism the oscillators would drift out of phase. Our knowledge of the molecular interactions governing the coupling interactions is ever increasing, however, with so many molecular components at play, and additional complications, such as the influence of the coupling on the clock frequency itself, it is challenging to construct a predictive molecular model. As such, in this study we have considered a coarsegrained model of the coupling mechanism in which it is assumed that oscillators can be adequately described by their relative phases.

By considering a relatively simple mechanism of coupling we can probe how biologically motivated perturbations can influence synchrony in an environment such as the PSM. For example, we consider the influence of mitosis, where cells temporarily pause their somitogenesis clock, on synchrony and demonstrate that the ratio of time spent in mitosis to the somitogenesis period can influence global synchrony in a physiologically relevant parameter regime.

There is a posteriorly increasing cell motility gradient along the AP axis in chick and it has previously been suggested that random cell motion, and hence exchange of local neighbours, could play an important role in maintaining synchronisation in the posterior PSM. We introduced cell motion into the posterior PSM by allowing cells to swap positions with their nearest neighbours with some probability, $p$, 
which can be related to measurements of mean-squared displacements of cells in the posterior PSM. Our simulation results indicate that neighbour swapping does indeed promote synchrony in the PSM.

In this study the local coupling of neighbouring oscillators is modelled using an on-lattice CA framework. Whilst the CA was chosen as it allows us to trivially simulate random cell movements via neighbour exchanges, with the degree of mixing within the population quantified by a single parameter, we note that alternative frameworks, such as the cellular Potts, vertex or off-lattice models, could be used to investigate the effects of mitosis block and random movement on oscillator synchronisation. While we expect that the simulation results are independent of the particular cell-level model under consideration, this touches on the general issues of what is the appropriate cell-based framework in which to model such phenomena and do different frameworks give different results? This problem is difficult to investigate as one cannot always relate parameters in one model to those in another [21]. However, we note that in certain cases it is possible to compare coarsened versions of different models (e.g. [22]).

The theory of phase-coupled oscillators has been applied to a range of physical phenomena. A ubiquitous feature of spatially coupled phase oscillators is the existence of rich dynamical behaviours, such as spiral waves. In this study, motivated by the observation of spatially synchronous oscillations in the posterior PSM, we have considered perturbations about the spatially synchronous solutions and examined how various biologically motivated perturbations can perturb the steady state. This approach allows us to focus on the effect of the perturbations rather than concern ourselves with the more complex, but not observed in the PSM, dynamical solutions to the phase equations.

Acknowledgement PJM and REB acknowledge the support of the Engineering and Physical Sciences Research Council through an EPSRC First Grant to REB. PKM was partially supported by a Royal Society-Wolfson Research Merit Award.

\section{References}

1. Jiang YJ, Aerne BL, Smithers L, Haddon C, Ish-Horowicz D, Lewis J (2000) Notch signalling and the synchronization of the somite segmentation clock. Nature 408(6811):475-479

2. Rida PCG, Le Minh N, Jiang YJ (2004) A Notch feeling of somite segmentation and beyond. Dev Biol 265(1):2-22

3. Horikawa K, Ishimatsu K, Yoshimoto E, Kondo S, Takeda H (2006) Noise-resistant and synchronized oscillation of the segmentation clock. Nature 441(7094):719-723

4. Mara A, Schroeder J, Chalouni C, Holley SA (2007) Priming, initiation and synchronization of the segmentation clock by delta D and delta C. Nat Cell Biol 9(5):523-530

5. Mara A, Schroeder J, Holley SA (2008) Two delta C splice-variants have distinct signaling abilities during somitogenesis and midline patterning. Dev Biol 318(1):126-132

6. Bénazéraf B, Francois P, Baker RE, Denans N, Little CD, Pourquié O (2010) A random cell motility gradient downstream of FGF controls elongation of an amniote embryo. Nature 466(7303):248-252 
7. Uriu K, Morishita Y, Iwasa Y (2010) Random cell movement promotes synchronization of the segmentation clock. Proc Natl Acad Sci U S A 107(11):4979

8. Mara A, Holley SA (2007) Oscillators and the emergence of tissue organization during zebrafish somitogenesis. Trends Cell Biol 17(12):593-599

9. Baker RE, Schnell S, Maini PK (2008) Mathematical models for somite formation. Curr Top Dev Biol Vol. 81 Chapter 6, 183-203

10. Goldbeter A, Pourquié O (2008) Modeling the segmentation clock as a network of coupled oscillations in the Notch. Wnt and FGF signaling pathways. J Theor Biol 252(3):574-585

11. Hirata H, Yoshiura S, Ohtsuka T, Bessho Y, Harada T, Yoshikawa K, Kageyama R (2002) Oscillatory expression of the bHLH factor Hes1 regulated by a negative feedback loop. Science 298(5594):840-843

12. Lewis J (2003) Autoinhibition with transcriptional delay: a simple mechanism for the zebrafish somitogenesis oscillator. Curr Biol 13(16):1398-1408

13. Rodríguez-González JG, Santillán M, Fowler AC, Mackey MC (2007) The segmentation clock in mice: interaction between the Wnt and Notch signaling pathways. J Theor Biol 248(1):37-47

14. Santillan M, Mackey MC (2008) A proposed mechanism for the interaction of the segmentation clock and the determination front in somitogenesis. PLoS One 3(2):e1561

15. Morelli LG, Ares S, Herrgen L, Schröter C, Jülicher F, Oates AC (2009) Delayed coupling theory of vertebrate segmentation. HFSP J 1(3):55-66

16. Murray PJ, Maini PK, Baker RE (2011) The clock and wavefront model revisited. J Theor Biol 283(1):227-238

17. Riedel-Kruse IH, Muller C, Oates AC (2007) Synchrony dynamics during initiation, failure, and rescue of the segmentation clock. Science 317:1911-1915

18. Kuramoto Y (1981) Rhythms and turbulence in populations of chemical oscillators. Physica A 106(1-2):128-143

19. Giudicelli F, Özbudak EM, Wright GJ, Lewis J (2007) Setting the tempo in development: an investigation of the zebrafish somite clock mechanism. PLoS Biol 5(6):e150

20. Kane DA (1998) Cell cycles and development in the embryonic zebrafish. Methods Cell Biol 59:11-26

21. Osborne JM, Walter A, Kershaw SK, Mirams GR, Fletcher AG, Pathmanathan P, Gavaghan D, Jensen OE, Maini PK, Byrne HM (2010) A hybrid approach to multiscale modelling of cancer. Phil Trans 368, 5013-5028

22. Murray PJ, Edwards CM, Tindall MJ, Maini PK (2009) From a discrete to a continuum model of cell dynamics in one dimension. Phys Rev E 80(3):031912-1-031912-10 